\title{
Variabilidad de la temperatura superficial del mar, identificación de surgencias costeras y su relevancia en un área marina costera protegida del desierto de Atacama, Chile ${ }^{1}$
}

\author{
Martín Farías Salvador ${ }^{2}$ y Consuelo Castro Avaria²
}

\begin{abstract}
RESUMEN
En el litoral de la III Región de Atacama, Chile, a partir de imágenes satelitales se estudió la variabilidad espacio temporal de la Temperatura Superficial del Mar (TSM) y sus anomalías. Se utilizó el transporte de Ekman para estimar la actividad de surgencias costeras, y debido a que el área de estudio se encuentra cercana al límite norte de la convergencia subtropical, se analizó su homogeneidad de acuerdo a las variables descritas. Los análisis se efectuaron para un período de tres años consecutivos, los cuales presentaron condiciones océanoatmosféricas opuestas que corresponden al desarrollo de un evento frío "La Niña 1996-97" y de un evento cálido "El Niño 1997-98".

Se concluye que si bien toda el área de estudio sigue tendencias estacionales e interanuales similares, se encuentran zonas en que la variabilidad que presentan la TSM, las anomalías y la actividad de surgencias es diferente. Como el transporte de Ekman utilizado contiene datos en una escala espacial global, se propone un índice de surgencia y un método digital para localizar sus focos, en una escala local.
\end{abstract}

Palabras clave: TSM, surgencia costera, Transporte de Ekman, Áreas marinas y costeras protegidas.

\begin{abstract}
In the coastal zone of the Atacama Region, Chile, through satellite images the variability of the Sea Surface Temperature (SST) and its anomalies were studied. To estimate the coastal upwelling activity, the Ekman transport was used and since the study area is near the northern limit of the subtropical convergence, with the above mentioned data an analysis was performed on the homogeneity of the whole area. The analysis were done for a period including three consecutive years, which present different oceanic and atmospheric conditions, a cold event "La Niña 1996-97" and a warm event "El Niño 1997-98".

Conclusions show that although the whole area follows seasonal and inter annual similar trends, there are zones in which the variability of the SST, its anomalies and the Ekman transport are different. As the Ekman transport used is a global index, a local index and a digital method to detect upwelling foci are proposed.
\end{abstract}

Key words: SST, coastal upwelling, Ekman transport, Protected marine and coastal areas.

1 Artículo recibido el 6 de junio de 2008 y aceptado el 1 de octubre de 2008.
2 Instituto de Geografía, Pontificia Universidad Católica de Chile (Chile).E-mail: mfariass@uc.cl; consuelo@uc.cl 
En la actualidad el borde costero de algunas regiones de Chile soporta una gran presión por el uso de sus recursos. En dicho espacio, conformado por un ámbito marino y uno terrestre, se manifiestan variados conflictos por incompatibilidad de algunos usos antrópicos con la naturaleza y singularidad del borde costero. Entre los principales problemas ambientales de la zona costera chilena destacan los vertidos domésticos, industriales y mineros líquidos sin depurar; la sobreexplotación de algunos recursos vivos; y el desarrollo inmobiliario en algunas regiones, provocando la fragmentación y disminución de la superficie de hábitats costeros críticos como las dunas litorales y zonas húmedas (Barragán et al., 2004).

Junto al patrimonio natural del litoral de Atacama, es preciso destacar que un $70 \%$ de los terrenos costeros de la III Región son de propiedad fiscal; en consecuencia, las investigaciones que aporten al conocimiento $y$ puesta en valor de dicho espacio marítimoterrestre, contribuirán a orientar los usos idóneos compatibles con la conservación de las áreas marino costeras de mayor relevancia. Respecto del manejo de Parques Marinos y Costeros, Salm et al. (2000) destacan que el uso sustentable de los recursos de las áreas litorales requiere que algunas zonas se resguarden en el estado más cercano a su condición natural, salvaguardando hábitats y paisajes de importancia funcional y escénica del litoral. En Chile, la necesidad e importancia de la creación de una Red de Parques y Reservas Marinas es analizada por Fernández \& Castilla (2005).

En este litoral las aguas marinas frías y ricas en nutrientes sustentan ecosistemas marinos de importancia para la conservación y la preservación de la biodiversidad marinocostera mundial. Lo anterior, sumado a la inexistencia de fuentes terrestres contaminantes, determinó que en el año 2005 se creara el Área Marino Costera Protegida de Múltiples Usos (AMCP-MU) Isla Grande de Atacama (CONAMA-PNUD, 2006). El borde costero posee un variado y atractivo patrimonio natural y cultural; además de la riqueza de recursos marinos y condiciones de accesibilidad que presenta la franja de playas, escollos rocosos y terrazas marinas, que han sustentado algunas actividades humanas desde tiempos prehistóricos (Castro et al., 2006).

Las surgencias o afloramientos de aguas marinas corresponden a movimientos ascendentes mediante los cuales las aguas localizadas a profundidades generalmente menores de 100-200 metros son Ilevadas hacia la superficie; con ello se produce un aporte de nutrientes a la zona fótica, posibilitando la fotosíntesis y en consecuencia, la fertilización de las aguas superficiales empobrecidas por el consumo biológico.

A escala general, en todos los océanos las áreas más productivas son aquellas localizadas próximas a las costas occidentales de los continentes donde ocurren las surgencias costeras; así, la distribución espacial de la productividad mundial de los océanos muestra que alrededor del $75 \%$ de la cosecha comercial global se efectúa en las áreas costeras con surgencias y que, en el océano abierto ocurre alrededor del 25\% de dicha cosecha (SHOA, 1996).

El origen de las surgencias costeras se relaciona con la dirección y fuerza del viento sobre la superficie del mar y con la orientación de la línea de costa. Por efecto de Coriolis, las aguas marinas próximas a la costa son removidas desde el área de transporte por el flujo horizontal, desplazadas hacia el océano abierto y desviadas hacia la izquierda en el hemisferio sur, lo que se conoce como transporte de Ekman. Por este mecanismo asciende agua de reemplazo, la cual proviene de niveles más profundos y aporta nutrientes a la superficie oceánica (Dever, 1997; Alvarinho \& Kawamura, 2004; Chelton et al., 2007).

Debido a su importancia, en diversas zonas del mundo los eventos de surgencia se han estudiado en sus distintos aspectos, tales como: los nutrientes que aportan a la zona fótica (Silva y Valdenegro, 2003; Bode et al., 2006; Wilkerson et al., 2006), la productividad generada por estos nutrientes (Marín y Olivares, 1999; Barbieri et al., 1995; Daneri et al., 2000; Figueiras et al., 2002; Grob et al., 2003; Wilkerson et al., 2006; Montecino et al., 2006; McManus et al., 2007) y como consecuencia, la sustentación de una importante cadena trófica mari- 
na, no solamente en donde se producen estos eventos, sino también en aguas adyacentes a ellos (Cole \& McGlade, 1998; Escribano et al., 2001; Figueiras et al., 2002; Piñones et al., 2007; Medina et al., 2007).

La posición geográfica de Chile favorece la generación de surgencias costeras, debido a la orientación Norte-Sur de la costa y a la dirección de los vientos predominantes soplando desde el sur y el suroeste. Fonseca y Farías (1987), y posteriormente Cáceres y Arcos (1991), señalaron la presencia de focos frecuentes de surgencia en Antofagasta y Mejillones; Iquique; Coquimbo; Valparaíso y Talcahuano; determinando que un evento de surgencia es un fenómeno local y esporádico, con una duración típica de una semana, que ocurre en respuesta a la intensidad y persistencia de los vientos locales de corta duración.

Con los factores mencionados que favorecen la existencia de surgencias y agregando la presión atmosférica, se genera el índice de surgencia de Ekman (Ruttlant y Montecino, 2002; Zaytsev et al., 2003; Ekman en Marchesiello \& Estrade, 2007; Middleton et al., 2007), el que posibilita estimar la actividad de surgencia en épocas pasadas, a mesoescala, gracias a series de tiempo de los datos involucrados, los que por largo tiempo se han estado recolectando. Además, una de las formas de detectar las zonas de surgencias y sus focos, posibilitando el estudio de su variabilidad espaciotemporal, ha sido mediante mediciones de la temperatura superficial del mar (TSM) con termómetros in situ y buques oceanográficos. En la actualidad y gracias al avance tecnológico, se cuenta con la capacidad de inferencia de esta variable desde datos captados por sensores remotos a bordo de satélites, los que permiten abarcar grandes áreas con una frecuencia incluso horaria (Fonseca y Farías, 1987; Cáceres y Arcos, 1991; Cole \& McGlade, 1998; Castro et al., 2006; Zavala-Hidalgo et al., 2006; Lazarus et al., 2007).

Dada la importancia de la región se hace necesario conocer mejor la variabilidad térmica superficial del mar en la zona donde se encuentra inserta el área protegida de Isla Grande de Atacama, durante el desarrollo de un evento frío "La Niña 1996-97", y de un evento cálido "El Niño 1997-98". Mediante la utilización de imágenes satelitales y del transporte de Ekman se analiza la variabilidad de TSM y sus anomalías, para detectar las zonas y focos de surgencia. Finalmente se propone un índice local de surgencia, basado en el transporte de Ekman, TSM y sus anomalías.

\section{Área de estudio}

El litoral de Atacama (Figura $N^{0} 1$ ) se encuentra cercano al límite norte de la línea de convergencia subtropical (Fuenzalida et al., 2007) y presenta condiciones favorables para la ocurrencia de surgencias costeras tales como: la forma y la orientación norte sur de la línea de costa; la dirección sur y suroeste de los vientos predominantes; una plataforma continental angosta y rocosa con islotes e islas como Ramada, Chata y Grande de Atacama; además de la pendiente del talud continental.

En el ámbito terrestre, las terrazas marinas sedimentarias son un rasgo relevante del

Figura $\mathrm{N}^{0} 1$

ÁREA DE ESTUDIO

Sector costero - Región de Atacama - Chile

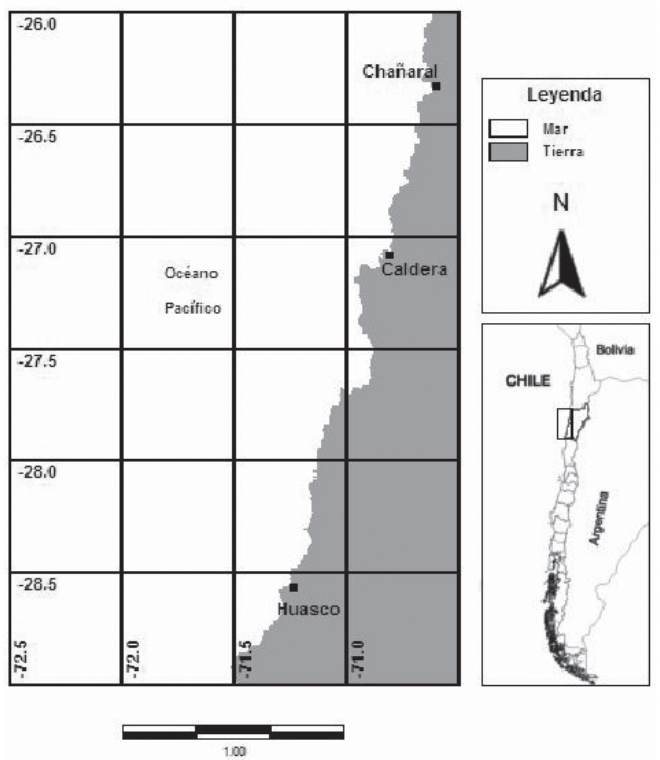

Fuente: Elaboración propia. 
relieve, particularmente entre Caldera y el río Copiapó. Así, en Bahía Inglesa las terrazas marinas de estratos fosilíferos dan cuenta de la historia paleogeográfica de la región. Los materiales de dichas terrazas son interpretados como sedimentos depositados en un ambiente marino sublitoral de plataforma continental de edad miocénica. La presencia de depósitos de fosforita, que son explotados en la actualidad, indican la existencia de un paleoocéano con surgencias de entre 45 y 180 metros de profundidad, las cuales, ricas en fosfatos y nutrientes generaron estos depósitos de la paleobahía de Caldera, en condiciones de un clima subtropical (Marquardt, 1999).

En la actualidad, las aguas de surgencia y de la corriente de Humboldt sustentan los ecosistemas marinos del Área Marino Costera Protegida de Múltiples Usos (AMCP-MU) Isla Grande de Atacama (CONAMA, PNUD, 2006), localizada entre Punta Morro (27 06' S) y la desembocadura del río Copiapó $\left(27^{\circ} 19^{\prime} \mathrm{S}\right)$. El área protegida comprende en su parte terrestre los terrenos de playa considerando 80 metros desde la línea de más alta marea; y en su parte oceánica, la columna de agua y los fondos marinos hasta media milla en el mar, incluyendo variados ecosistemas, además de las islas Chata y Grande de Atacama. Uno de los aspectos relevantes del AMCP-MU es que se orienta a conectar la conservación de espacios terrestres con los espacios marinos y que permite usos múltiples de los recursos naturales, principalmente para beneficio de las poblaciones locales.

\section{Materiales y métodos}

\section{Materiales}

El período de estudio corresponde a observaciones satelitales, registros de temperatura superficial del mar y del transporte de Ekman desde enero de 1996 a diciembre de 1998. Las imágenes satelitales de TSM son diarias, y tienen una resolución espacial de $1 \mathrm{~km}^{2}$; estas fueron facilitadas por la Empresa Pesquera Coloso S.A. Los promedios mensuales históricos de TSM fueron digitalizados del Atlas del Servicio Hidrográfico y Oceanográfico de la Armada (SHOA, 1996) e interpolados para llevarlos a la misma resolución es- pacial de las imágenes. Asimismo, se usó un modelo de elevación digital generado de cartas topográficas escala 1:250.000 provenientes del Instituto Geográfico Militar (IGM), y los promedios mensuales de transporte de Ekman calculados para una resolución espacial de un grado de latitud/longitud en el servidor de la División de Investigación Ambiental (ERD, 2008) de NOAA (National Oceanographic and Atmospheric Administration) de los Estados Unidos. Todos los datos e imágenes fueron georreferenciados de acuerdo al elipsoide y datum WGS84.

\section{Metodología}

Para determinar si toda el área de estudio presenta una variabilidad homogénea, esta se dividió en tres zonas que abarcan cada un grado de latitud; así se reconocen: zona norte (de $26^{\circ}$ a $\left.27^{\circ} \mathrm{S}\right)$, zona centro $\left(27^{\circ}\right.$ a $\left.28^{\circ} \mathrm{S}\right)$ y zona sur $\left(28^{\circ}\right.$ a $\left.29^{\circ} \mathrm{S}\right)$ (Figura $\left.\mathrm{N}^{\circ} 1\right)$. A partir de las imágenes se realizaron diversos cálculos para la determinación de parámetros que se indica a continuación:

\section{Promedios mensuales de TSM}

Para el cálculo se eliminaron todas aquellas imágenes diarias que presentaron más de un $50 \%$ de cobertura nubosa, especialmente en lugares más próximos a la costa. Enseguida, se procedió a calcular promedios mensuales para comparar la variabilidad de TSM entre zonas.

\section{Promedios mensuales de anomalías}

Utilizando los promedios mensuales calculados y los promedios mensuales históricos de TSM, descritos anteriormente, se determinaron los promedios mensuales de anomalía y también, una comparación de variabilidad entre zonas.

\section{Transporte de Ekman}

Se utilizó el modelo de elevación digital (datos de relieve) para obtener la orientación de la línea de costa cada 1 kilómetro y sacar un promedio de orientación para cada una de las tres zonas. Con esta información se procedió a calcular el transporte de Ekman mensual para cada una de ellas y se realizó una comparación entre zonas. 
Zonas de surgencia escala local

Como el transporte de Ekman es un índice de surgencia global (un grado de lat./ long.) se diseñó un índice de surgencia local (cada 1 kilómetro). Previamente se estandarizaron los datos de TSM, de anomalías y de transporte para compatibilizarlos y poder generar el índice propuesto. Este último consistió en el transporte de Ekman estandarizado, dividido por la suma estandarizada de la TSM y la anomalía. Con lo anterior se obtiene un índice en valores relativos que se clasificó en alto, medio, bajo y muy bajo.

\section{Focos de surgencia escala local}

Para identificar los focos de surgencia, a escala local, se utilizó nuevamente el modelo de elevación digital con el propósito de determinar una línea de costa conteniendo los 2 primeros kilómetros de mar. Esta línea de costa marina se parcializó en muestras latitudinales cada 1 kilómetro y para cada uno de ellas se calculó la TSM promedio de los 36 meses, con lo que se determinaron los lugares costeros que representan donde se inician los eventos de surgencias.

Los resultados de esta etapa y de la anterior fueron confrontados con un análisis visual de 100 imágenes seleccionadas aleatoriamente.

\section{Resultados y discusión}

Imágenes satelitales utilizadas

Las imágenes satelitales presentaron una gran cobertura de nubes, seleccionándose 120 imágenes para 1996, 144 para 1997 y 83 para 1998. En las Figuras 2a, 2 b y 2c se observa la distribución de la TSM con una menor cobertura de nubes, para tres temporadas de diferentes características: un evento La Niña (enero 1996); un periodo de transición (enero 1997) y, un evento El Niño (enero 19998), respectivamente.

Figura $\mathrm{N}^{\circ} 2$

DISTRIBUCIÓN SUPERFICIAL DE TEMPERATURA ( ${ }^{\circ} \mathrm{C}$ ) PARA A) 20/01/1996, B) 20/01/1997 Y C) 14/01/ 1998. LA ESCALA DE TSM SE MUESTRA EN LA PARTE INFERIOR DEL PANEL CENTRAL

TSM $\left({ }^{\circ} \mathrm{C}\right)$ 20-01-1996

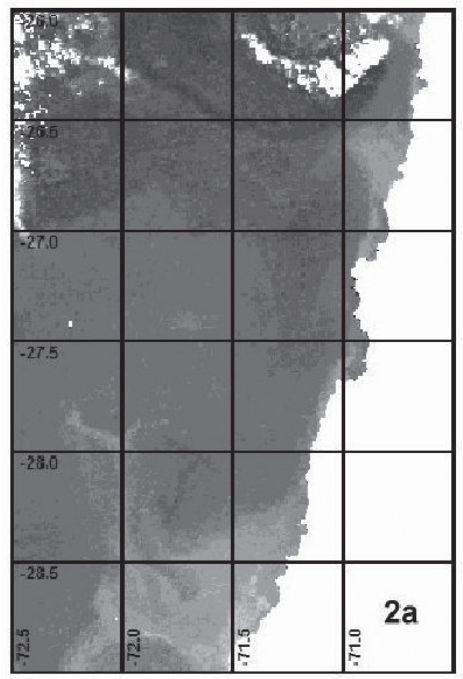

$\operatorname{TSM}\left({ }^{\circ} \mathrm{C}\right)$ 20-01-1997

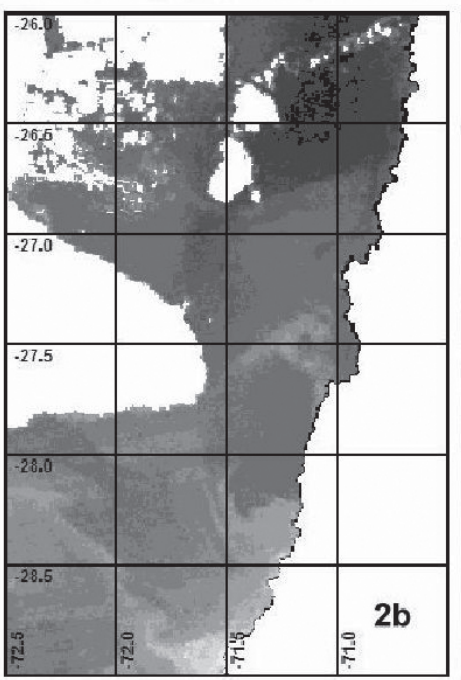

莺
$\operatorname{TSM}\left({ }^{\circ} \mathrm{C}\right) 14-01-1998$

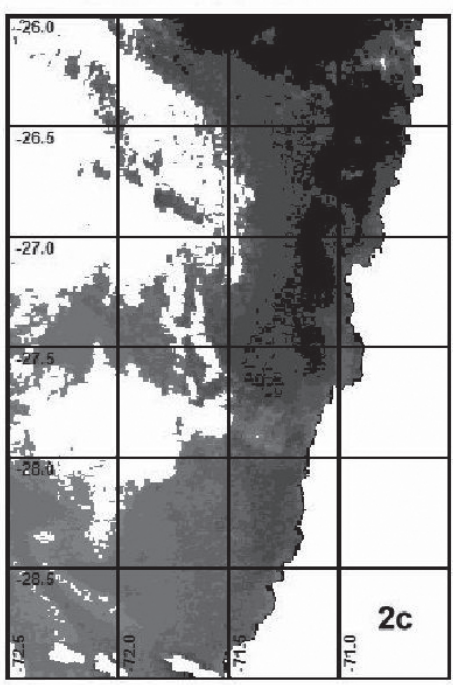

Fuente: Elaboración propia. 


\section{Variabilidad de TSM}

De acuerdo a los promedios mensuales de TSM (Figura $N^{\circ} 3$ ), las tres zonas se manifiestan de acuerdo al ciclo anual conocido, frío en invierno y temperado en verano. Además, se presenta una tendencia latitudinal, también conocida, en que las zonas más al norte siempre muestran diferencias positivas sobre las zonas más al sur. Respecto a la variabilidad interanual, ya en enero de 1997 se aprecia un aumento de TSM en comparación al mismo mes de 1996, esta diferencia se va incrementando en los meses de otoño y tiene su máxima diferencia en los meses de invierno de 1997. De ahí en adelante la diferencia comienza a decaer lentamente y finalmente en marzo-abril de 1998 prácticamente desaparece.

\section{Diferencias de TSM entre zonas}

Para detectar si existen diferencias en la variabilidad de TSM entre zonas, se grafican las diferencias entre las zonas norte y centro, junto a diferencias entre las zonas norte y sur (Figura $N^{\circ} 4$ ). Si la variabilidad de TSM se debiera exclusivamente a la ubicación latitudinal, las diferencias entre zonas debieran permanecer relativamente constantes. Sin embargo, se aprecian notorias variaciones en dichas diferencias, especialmente entre las zonas norte y sur en que sus amplitudes son mayores. Para los tres años se aprecia que las diferencias se incrementan en los meses de verano. En el resto de los meses de 1996 las diferencias son menores y parejas (excepto junio), sin embargo, los mismos meses de los años 1997 y 1998 presentan mayores variaciones en las respectivas diferencias. Para los 36 meses abarcados, la línea de tendencia de las diferencias entre las zonas norte y centro muestra una pequeña pendiente al aumento, mientras que aquella para las diferencias entre las zonas centro y sur es mayor.

De este análisis se puede inferir que al encontrarse el área de estudio cercana a la convergencia subtropical, la zona norte está más expuesta a las aguas subtropicales y la zona sur a las aguas subantárticas. Por lo anterior, en los periodos más cálidos se presentan mayores diferencias entre ambas zonas.

\section{Variabilidad de anomalías de TSM}

En la Figura $\mathrm{N}^{0} 5$ se indican las anomalías de TSM para todo el período de análisis. Las tres zonas muestran un período de anomalías negativas hasta febrero de 1997, las que posteriormente se transforman en anomalías positivas alcanzando su máximo en junio-julio de 1997, para decaer paulatinamente hasta abril de 1998 (excepto en diciembre de 1997, que presenta un aumento importante). Posterior al mes de abril de 1998 se presenta un nuevo aumento de anomalías positivas en junio del mismo año, para después retornar a anomalías negativas hasta el fin del periodo. De este análisis, se

Figura $N^{\circ} 3$

VARIABILIDAD DE TSM PARA LAS TRES ZONAS DURANTE LOS 36 MESES DEL PERIODO

(Promedios mensuales)

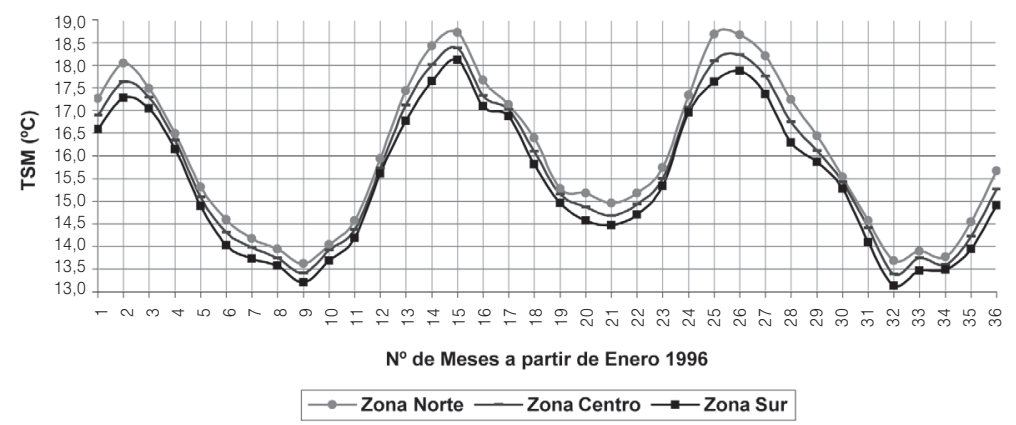

Fuente: Elaboración propia. 
Figura $N^{\circ} 4$

DIFERENCIAS ENTRE ZONAS DE LA VARIABILIDAD DE TSM PARA LOS 36 MESES

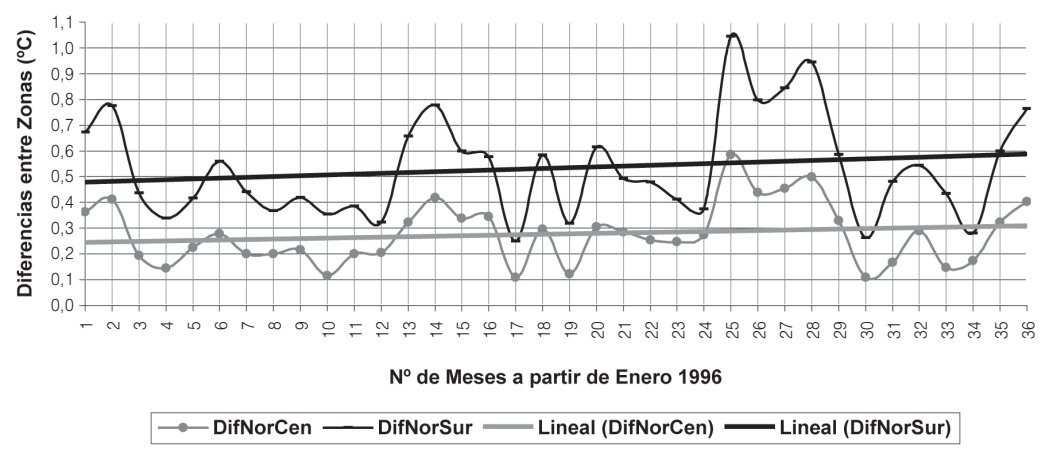

Fuente: Elaboración propia.

Figura $N^{0} 5$

VARIABILIDAD DE ANOMALÍAS DE TSM PARA LAS TRES ZONAS DURANTE LOS 36 MESES DEL PERIODO

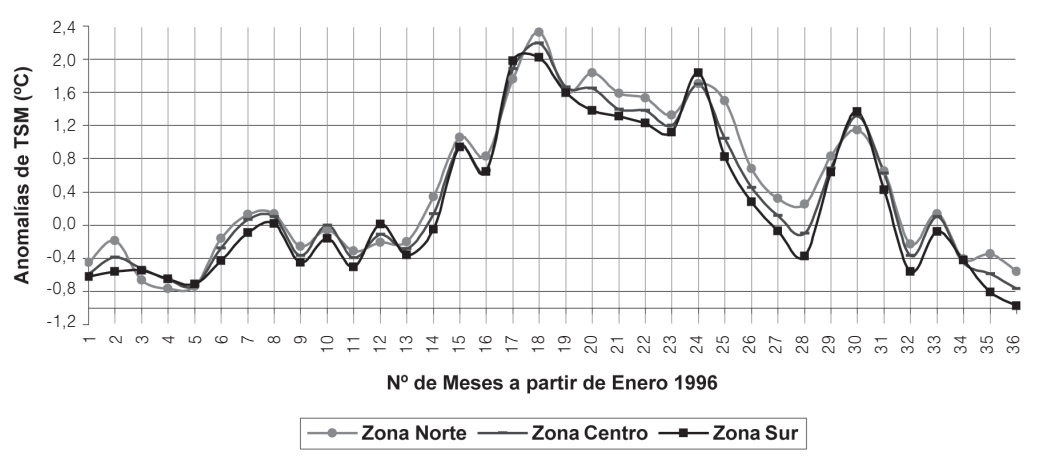

Fuente: Elaboración propia.

puede inferir claramente la influencia del evento "El Niño", determinando su efecto desde marzo-abril de 1997 hasta marzoabril de 1998, presentando una nueva "réplica" entre mayo y julio de este último año.

\section{Diferencias de anomalías de TSM entre zonas}

El análisis de diferencias de anomalías de TSM entre zonas (Figura $\mathrm{N}^{\circ}$ 6) revela resultados muy parecidos al de diferencias de TSM. En general, la zona norte tiene mayores anomalías positivas que las zonas centro y sur, tal como se observa en las líneas de tendencia. Estas últimas poseen una pendiente positiva en el período observado, lo que significa que las diferencias son menores en eventos fríos y aumentan en eventos cálidos. No obstante, en este gráfico apreciamos varios meses del período en que la zona norte presenta menores anomalías positivas que las zonas centro y sur; es el caso de marzo, abril y diciembre de 1996; mayo y diciembre de 1997, y junio de 1998. Esto permite inferir que al interior del área de estudio hay diferencias notorias en la variabilidad térmica superficial del mar. 
Figura $\mathrm{N}^{\circ} 6$

DIFERENCIAS ENTRE ZONAS DE LA VARIABILIDAD DE ANOMALÍAS DE TSM PARA LOS 36 MESES

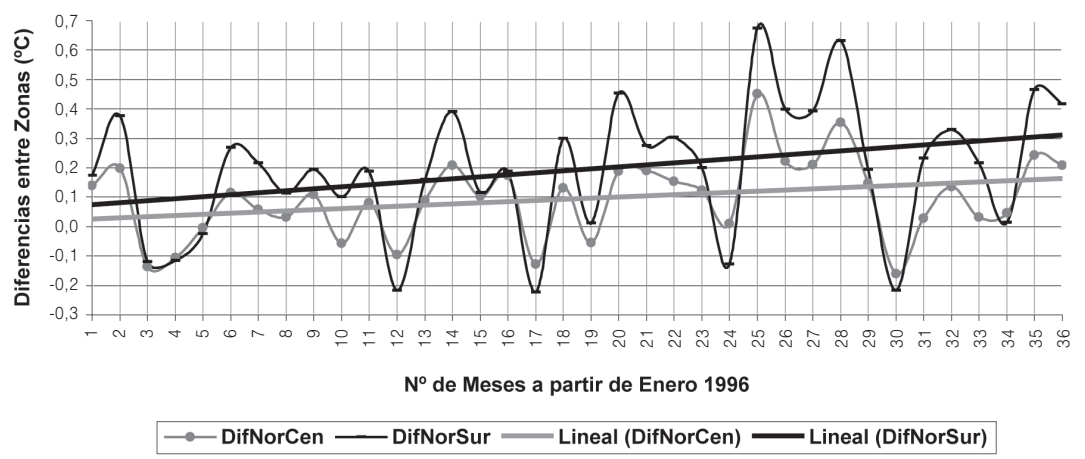

Fuente: Elaboración propia.

\section{Transporte de Ekman}

El transporte de Ekman, expresado en la Figura $N^{0} 7$, muestra la existencia en todo el litoral de la III Región de Atacama de factores que inciden positivamente en la presencia y actividad de surgencias, especialmente en aquellos eventos fríos como "La Niña". En general, las mayores condiciones para la ocurrencia de surgencias se dan en los meses de primavera y verano, y las menores condiciones en invierno. A esto se suma que la variabilidad interanual está muy influen- ciada por el efecto del evento "El Niño". Así, se observa en la Figura $N^{\circ} 7$ que el año 1996 alcanza los índices más altos con un promedio anual de $205 \mathrm{~m}^{3} / \mathrm{seg} 100 \mathrm{~m}$; 1997 con un promedio de anual de $114 \mathrm{~m}^{3 /}$ seg $100 \mathrm{~m}$. y 1998 con un promedio anual de $135 \mathrm{~m}^{3} / \mathrm{seg} 100 \mathrm{~m}$.

Diferencias de transporte de Ekman entre zonas

El transporte de Ekman calculado, en general sigue ciclos estacionales e interanua-

Figura $\mathrm{N}^{0} 7$

VARIABILIDAD DEL TRANSPORTE DE EKMAN PARA LAS TRES ZONAS DURANTE

LOS 36 MESES DEL PERIODO

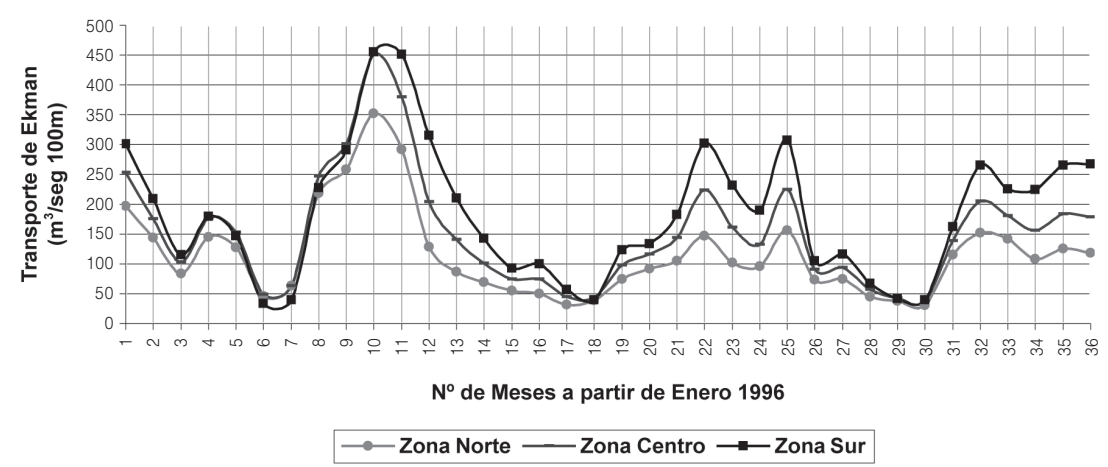

Fuente: Elaboración propia. 
les similares para todas las zonas y, como se aprecia en la Figura $N^{\circ}$ 8, las diferencias entre zonas son notorias. La zona sur del área de estudio presenta durante los 36 meses valores mayores (valores negativos en la Figura $\mathrm{N}^{\circ}$ 8), excepto en junio-julio de 1996. Las diferencias aumentan especialmente en los meses de primavera y verano, para decrecer totalmente en los meses de invierno. En el período abarcado, la línea de tendencia de diferencias entre la zona norte y centro se muestra estable con una pendiente casi nula, no así la línea respectiva para las diferencias entre las zonas norte y sur, con una pendiente negativa importante.

\section{Zonas de surgencia escala local}

El índice local de surgencia propuesto fue calculado para los 36 meses contemplados y su resultado se exhibe en la Figura $\mathrm{N}^{\circ}$ 9. Sus valores van entre 0 (menor índice) y 178 (mayor índice). Como se aprecia, la zona sur presenta los mayores valores, la zona centro valores intermedios y la zona norte los menores valores. Esto fue corroborado por un análisis visual que se realizó en 100 imágenes aleatorias, el que consistió en determinar la frecuencia de ocurrencia de eventos de surgencia y cuyo resultado demostró que la frecuencia de la zona sur es 4 veces mayor que la frecuencia de la zona norte. Todo lo anterior está acorde con las variables anteriormente analizadas, en que se establece que la zona sur está menos ex-
Figura $N^{\circ} 9$

ZONAS DE SURGENCIA DETERMINADAS CON EL ÍNDICE LOCAL. EL ANCHO DE LÍNEA NEGRA, SUPERPUESTA PERPENDICULARMENTE A LA LÍNEA DE COSTA, REPRESENTA EL VALOR DEL ÍNDICE DE SURGENCIA, A MAYOR ANCHO MAYOR EL ÍNDICE

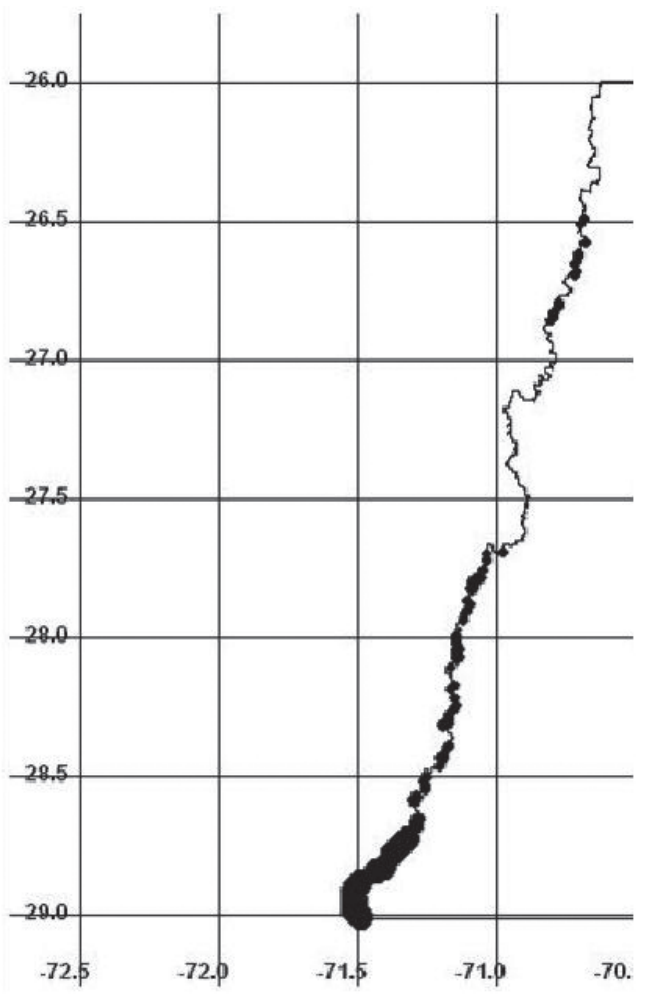

Fuente: Elaboración propia.

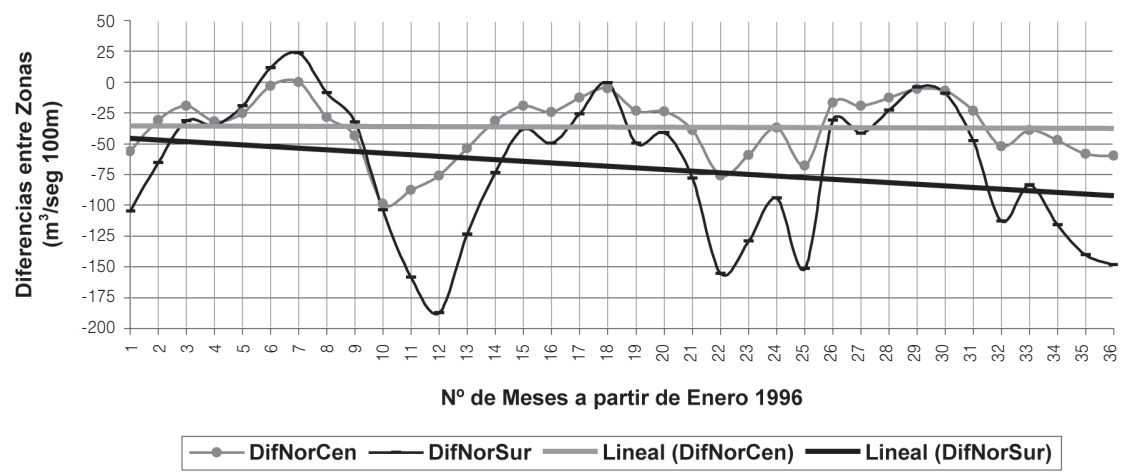

Fuente: Elaboración propia. 
puesta a las aguas subtropicales y, por lo tanto, presenta una mayor resistencia cuando eventos cálidos tienden a inhibir las surgencias.

\section{Focos de surgencia escala local}

Independientemente de la mayor o menor actividad de surgencia, es particularmente relevante conocer la localización de los focos de aguas surgentes en el Área Marino Costera Protegida de Múltiples Usos (AMCP-MU) Isla Grande de Atacama. En la Figura $N^{\circ} 10$, se distinguen, en base a TSM, los promedios de los 36 meses para la línea de costa marina, identificando los focos como la menor temperatura entre sus áreas adyacentes.

Dentro del AMCP-MU considerada se detectaron 4 focos de surgencia como los más importantes, tanto con el índice local de surgencia como con el procedimiento recién descrito. Los focos, de norte a sur, son los siguientes: Punta Morro $(-27,120 \mathrm{~S}$ y $70,938 \mathrm{~W})$ con un índice de 140, Punta Medio $(-27,170 \mathrm{~S}$ y $-70.963 \mathrm{~W})$ con un índice de 145, Punta Totoral $(-27,229$ S y -70.955) con un índice de 135 y Punta Vial $(-27,254$ S y -70.955) con un índice de 135.

\section{Consideraciones finales}

Los análisis efectuados demuestran gran potencialidad de ocurrencia de surgencias en el litoral de la III Región de Atacama. La localización de este litoral, en una zona de transición entre el norte y centro del país y que además se encuentra cercana a la convergencia subtropical, determina que las aguas litorales y oceánicas son muy adecuadas para el análisis y seguimiento de eventos La Niña y El Niño.

De las variables consideradas en el análisis, es relevante el transporte de Ekman como indicador de la temporalidad de condiciones favorables para la ocurrencia de surgencias. Las mayores condiciones se dieron los meses de primavera y verano, y las menores condiciones en invierno. Además, la variabilidad interanual estuvo muy influenciada por el efecto del evento "El Niño".

Se encontraron diferencias de TSM y anomalías entre zonas, explicables por su posición latitudinal con valores decrecientes de norte a sur. Ante todo, las notorias diferencias de variabilidad de estos factores entre zonas se interpretan como la influencia de condiciones ambientales que producen oscilaciones en las masas de agua convergentes (aguas subtropicales y aguas subantárticas) en las cercanías del área de estudio.

El índice local de surgencias propuesto mostró que la zona sur presenta mayor actividad de surgencias con respecto a la zona norte. Lo anterior es concordante con los resultados entregados por los análisis de las

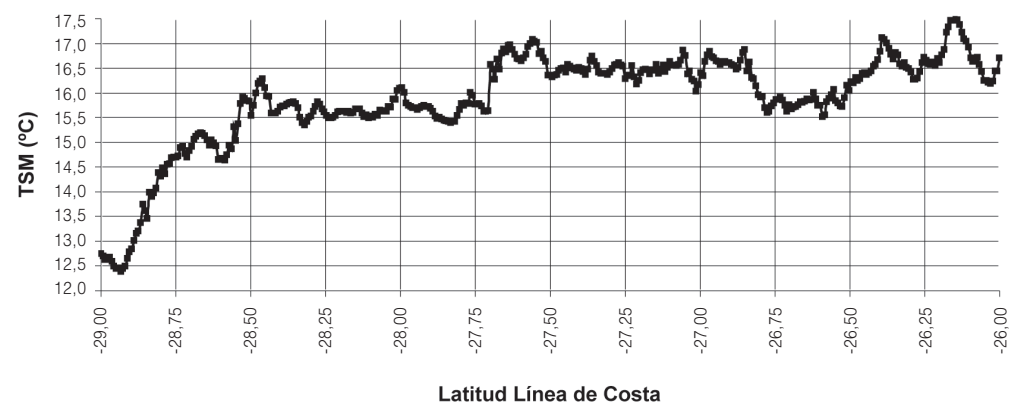

Fuente: Elaboración propia. 
otras variables consideradas en el estudio. Se agrega que el índice local de surgencias permite un mayor detalle espacial con respecto al transporte de Ekman utilizado, el cual opera en una escala global.

Por un lado, el método para detectar los focos de surgencia en forma digital facilita el procesamiento de gran número de imágenes satelitales. Por otro, su detalle espacial permite localizar los focos de surgencia con precisión de $1 \mathrm{~km}$. Esto adquiere mayor relevancia ya que las áreas marinas costeras protegidas de múltiples usos se orientan a conectar la conservación de espacios terrestres con los espacios marinos y mientras más detallada, espacialmente, la información que se obtenga, mejor contribuye a la toma de decisiones de uso del borde costero.

Dentro del AMCP-MU se detectaron 4 importantes focos de surgencia frente a puntas rocosas del litoral, los que han sido localizados con $1 \mathrm{~km}$ de precisión. Se espera que esta información sea de utilidad en la toma de decisiones del manejo marino y costero de esta área, especialmente con respecto a la protección de los focos de surgencia ante la eventual intervención de origen antrópico en el borde costero.

*Los autores agradecen a la Empresa Pesquera Coloso S. A. que facilitó las imágenes satelitales que posibilitaron este estudio, a la División de Investigación Ambiental de la organización NOAA de Estados Unidos por facilitar en su sitio WEB los cálculos de transporte de Ekman y al alumno Miguel Jara Meneses del Instituto de Geografía, quien confeccionó algunas de las figuras que ilustran este texto.

**Este artículo es parte de los resultados del Proyecto Fondecyt No 1070442. "Bases geomorfológicas para la determinación de áreas de conservación en terrenos fiscales del litoral de Atacama, III Región".

\section{Referencias bibliográficas}

ALVARINHO, J. L. \& KAWAMURA, H. Air-sea interaction, coastal circulation and primary production in the eastern Arabian Sea: a review. Journal of Oceanography, 2004, Vol. 60, p. 205-218.
BARBIERI, M. A.; BRAVO, M.; FARÍAS, M.; GONZÁlEZ, A.; PIZARRO, O. y YÁÑEZ, E. Fenómenos asociados a la estructura térmica superficial del mar observados a través de de imágenes satelitales en la zona norte de Chile. Investigaciones Marinas, 1995, Vol. 23, p. 99-122. Disponible en Internet: http://www.scielo.cl/scielo.php?pid=S0717$71781995002300007 \&$ script=sci_arttext

BARRAGÁN, J. M.; CASTRO, C. y ALVARADO, C. La zona costera chilena: el medio natural y el manejo integrado. Rev. Universidad Católica de Valparaíso, 2004, No 35, p. 17-35.

BODE, A.; ÁlVAREZ-OSORIO, M. T. \& VARELA, M. Phytoplankton and macrophyte contributions to littoral food webs in the galician upwelling estimated from stable isotopes. Marine Ecology Progress Series, 2006, Vol. 318, p. 89-102. Disponible en Internet: http://www.int-res.com/articles/meps_oa/ m318p089.pdf

CÁCERES, M. y ARCOS, D. Variabilidad de la estructura espacio temporal de un área de surgencia frente a la costa de Concepción, Chile. Rev. Investigación Pesquera, 1991, No36, p. 27-38.

CASTRO, C.; FARÍAS; M. y JARA, M. Variabilidad espacio temporal de las surgencias en el litoral de Atacama. En: CONAMA. Actas Primer Seminario Internacional de Áreas marinas y costeras protegidas. Caldera: CONAMA, 2006, Inédito.

CHELTON, D.; SCHLAX, M. \& SAMELSON, R. Summertime coupling between sea surface temperature and wind stress in the California current system. Journal of Physical Oceanography, 2007, Vol. 37, p. 495517.

COLE, J. \& MCGLADE, J. Clupeoid population variability, the environment and satellite imagery in coastal upwelling systems. Reviews in Fish Biology and Fisheries, 1998, Vol. 8, p. 445-471. Disponible en Internet: http://www.springerlink.com/content/ w7l5568264t52124/fulltext.pdf

CONAMA-PNUD. Conservación de la biodiversidad de importancia mundial a lo 
largo de la costa chilena. Áreas Marinas y Costeras Protegidas de Múltiples Usos: Isla Grande de Atacama Lafken Mapu Lahual. Santiago de Chile: Ocho Libros Editores, 2006.

DANERI, G.; DELLAROSA, V.; QUIÑONES, R.; JACOB, B.; MONTERO, P. \& ULLOA, O. Primary production and community respiration in the Humboldt Current System off Chile and associated oceanic areas. Mar, Ecol. Prog. Ser., 2000, Vol. 197, p. 41-49. Disponible en Internet: http:// www.int-res.com/articles/meps/197/ m197p041.pdf

DEVER, E. P. Wind-forced croos-shelf circulation on the northern California shelf. J. Phys. Oceanogr., 1997, Vol. 27, p. 15661571.

ERD. Southwest Fisheries Science Center. California: Environmental Research Division, 2008. Disponible en Internet: http:// swfsc.noaa.gov/ERD.aspx

ESCRIBANO, R.; MARÍN, V. \& HIDAL$\mathrm{GO}, \mathrm{P}$. The influence of coastal upwelling on the distribution of Canalus chilensis in the Mejillones Peninsula (northern Chile): Implications for its population dynamics. Hidrobiología, 2001, No 453/454, p. 143-151.

FERNÁNDEZ, M. \& CASTILLA, J. C. Marine conservation in Chile: Historical perspective, lessons and challenges. Conservation Biology, 2005, Vol. 19, $\mathrm{N}^{\circ}$ 6, p. 1752-1762.

FIGUEIRAS, F. G.; LABARTA, U. \& FERNANDEZ, M. J. Coastal upwelling, primary production and mussel growth in the Rias Baixas of Galicia. Hidrobiología, 2002, Vol. 484, p. 121-131.

FONSECA, T y FARÍAS, M. Estudio del proceso de surgencia en la costa chilena utilizando percepción remota. Rev. Investigación Pesquera, 1987, No 34, p. 33-46.

FUENZALIDA, R.; SCHNEIDER, W.; BLANCO, J. L.; GARCÉS, J. y BRAVO, L. Sistema de corrientes Chile-Perú y masas de agua entre Caldera e Isla de Pascua. Ciencia y Tecnología del Mar, Comité Oceanográfico
Nacional, 2007, Vol. 30, № 2, p. 5-16. Disponible en Internet: http://www.cona.cl/ctmol2/vol30-2/articulos/Fuenzalida/ fuenzalida.pdf

GROB, C.; QUIÑONES, R. y FIGUEROA, D. Cuantificación del transporte de agua costa-océano a través de filamentos y remolinos ricos en clorofila, en la zona centrosur de Chile $\left(35,5-37,5^{\circ} \mathrm{S}\right)$. Gayana, 2003, Vol. 67, N ${ }^{\circ} 1$, p. 55-67. Disponible en Internet: http://www.scielo.cl/pdf/gayana/v67n1/ art08.pdf

LAZARUS, S.; CALVERT, C.; SPLITT, M.; SANTOS, P.; SHARP, D.; BLOTMAN, P. \& SPRATT, S. Real time, high-resolution, space-time analysis of sea surface temperatures from multiple platforms. Monthly Weather Review, 2007, Vol. 135, p. 3158-3173. Disponible en Internet: http://www.srh. noaa.gov/mlb/PDFs/lazarus_etal_2007.pdf

MARQUARDT, C. Neotectónica de la franja costera y aportes a la geología regional entre Caldera y Caleta Pajonal $\left(27^{\circ} 00^{\prime}\right.$ $\left.27^{\circ} 45^{\prime} S\right)$, III Región de Atacama. Tesis de Magíster. Santiago de Chile: Departamento de Geología. Universidad de Chile, 1999.

MARCHESIELLO, P. \& ESTRADE, P. Eddy activity and mixing in upwelling systems: a comparative study of northwest Africa and California regions. Int. J. of Earth Sciences, 2007, In Press.

MARÍN, V. y OLIVARES, G. Estacionalidad de la productividad primaria en bahía Mejillones del Sur (Chile): una aproximación proceso-funcional. Rev. Chil. Hist. Nat., 1999, Vol. 72, p. 629-641.

MCMANUS, G.; COSTAS, B.; DAM, H.; LOPES, R.; GAETA, S. SUSUNI, S. \& ROSETTA, C. Microzooplankton grazing of phytoplankton in a tropical upwelling region. Hydrobiologia, 2007, Vol. 575, p. 69-81.

MONTECINO, V.; PAREDES, M. A. Y PAOLINI, P. Reinterpretando datos de clorofila en la costa centro-norte de Chile, considerando variabilidad ambiental de multiescala. Revista chilena de historia natural, 2006, Vol. 79, No 2, p. 213-223. Disponible 
en Internet: http://www.scielo.cl/scielo. php?pid=S0716-078X2006000200007 \&script $=$ sci_arttext

MEDINA, M.; ARANCIBIA, H. y NEIRA, S. Un modelo trófico preliminar del ecosistema pelágico del norte de Chile $\left(18^{\circ} 29^{\prime} \mathrm{S}\right.$ $\left.24^{\circ} 00^{\prime} \mathrm{S}\right)$. Investigaciones Marinas, 2007, Vol. 35, No 1, p. 25-38. Disponible en Internet: www.scielo.cl/pdf/imar/v35n1/art03.pdf

MIDDLETON, J.; VAN RUTH, P. \& WARD, T. El Nino effects and upwelling off south Austrália. Journal of Physical Oceanography, 2007, Vol. 37, No 10, p. 2458-2477.

PIÑONES, A.; CASTILLA, J. C.; GUÍÑ̃EZ, R. y LARGIER, J. L. Temperaturas superficiales en sitios cercanos a la costa en la bahía de Mejillones y centros de surgencia adyacentes. Ciencias Marinas, 2007, Vol. 33, $\mathrm{N}^{\mathrm{o}} 1$, p. 37-48.

RUTLLANT, J. y MONTECINO, V. Ciclos multiescala en el forzamiento de la surgencia y respuesta biológica em el centro-norte de Chile. Revista chilena de historia natural, 2002, Vol. 75, No 1, p. 217-231. Disponible en Internet: http://www.scielo.cl/scielo. php?script=sci_arttext\&pid=S0716078X2002000100020\&lng=pt\&nrm=iso

SALM, R.V.; CLARK, J. \& SIRIILA, E. Marine and Costal Protected Areas: A guide for planners and managers. Canadá: IUCN, 2000.
SILVA, N. y VALDENEGRO, A. Evolución de un evento de surgencia frente a punta Curaumilla, Valparaíso. Investigaciones Marinas, 2003, Vol. 31, № 2, p. 73-89. Disponible en Internet: http://www.scielo.cl/ scielo.php?script=sci_arttext\&pid=S0717$71782003000200007 \& \operatorname{lng}=\mathrm{es} \& \mathrm{nrm}=\mathrm{iso}$

SERVICIO HIDROGRÁFICO Y OCEANOGRÁFICO DE LA ARMADA DE CHILE (SHOA). Atlas Oceanográfico de Chile. Valparaíso: SHOA, 1996, Vol. 1.

WILKERSON, F.; LASSITER, A.; DUGDALE, A. \& HOGUE. V. The phytoplankton bloom response to wind events and upwelled nutrients during COOP WEST study. Deep-sea Research II, 2006, Vol. 53, NN 2526, p. 3023-3048.

ZAVALA-HIDALGO, J.; GALLEGOSGARCIA, A.; MARTÍNEZ-LÓPEZ, B.; MOREY, S. \& O'BRIEN, J. Seasonal upweIling on the western and southern shelves of the Gulf of Mexico. Ocean Dynamics, 2006, Vol. 56, p. 333-338.

ZAYTSEV, O.; CERVANTES-DUARTE, R.; MONTANTE, O. \& GALLEGOS-GARCÍA, A. Coastal upwelling activity on the pacific shelf of the Baja California Peninsula. Journal of Oceanography, 2003, Vol. 59, $\mathrm{N}^{\circ} 4$, p. 387-535. 
